\title{
Post-traumatic Stress Disorder in Facial Injuries: A Comparative Study
}

\author{
${ }^{1} \mathrm{NT}$ Prashanth, ${ }^{2} \mathrm{HP}$ Raghuveer, ${ }^{3} \mathrm{R}$ Dilip Kumar, ${ }^{4} \mathrm{ES}$ Shobha, ${ }^{5}$ Vinod Rangan, ${ }^{6}$ Baswaraj Hullale
}

\begin{abstract}
Aim: The aim of the study was to identify the presence of post-traumatic stress disorder (PTSD) in patients who had sustained facial injuries, additionally, we aimed to identify other variables that may modify the psychological response to trauma that include gender, age and presence of disfigurement posttreatment and visible scars/orthopedic injuries.
\end{abstract}

Materials and methods: Participants comprised of 460 patients from several multinodal trauma centers in Bengaluru City, Karnataka, who had sustained facial injuries that had healed either with or without significant disfigurement or scarring and with visible/orthopedic injuries. One hundred and eleven patients of the chosen 460 had sustained nondisfiguring facial injuries while 153 patients sustained disfiguring facial injuries, 64 patients who sustained facial injury (i.e. 19.5\%) were lost to follow-up and were not included in the study. One hundred and thirty-two had sustained orthopedic/visible injuries; however, in this group, 18 (i.e. 13.6\%) patients were lost to follow-up and were excluded from the study. The impact of events scale (IES) was used to check the presence of PTSD.

Results: Statistically significant higher means of IES were present in patients with disfiguring facial injuries compared to nondisfiguring facial injuries, female patients compared to male patients, patients with disfiguring facial injuries compared to orthopedic/visible injuries and patients who were younger than 50 years of age compared to patients who were older than 50 years of age and the results observed were similar at all three study intervals (date of discharge (DOD), 1 month and 6 months postoperatively).

Conclusion: Patients with disfiguring facial injuries had significantly higher PTSD levels compared to patients with nondisfiguring facial injuries, patients with orthopedic/visible injuries had statistically significant lower IES scores which could not be strictly termed PTSD when compared to patients with disfiguring facial injuries who had high scores of IES corresponding to high levels of PTSD and these results were observed at all three study intervals (DOD, 1 and 6 months postoperatively). Female patients with disfiguring facial injuries

\footnotetext{
${ }^{1-5}$ Department of Oral and Maxillofacial Surgery, Dayananda Sagar College of Dental Sciences, Bengaluru, Karnataka, India

${ }^{6}$ Department of Orthodontics and Dentofacial Orthopedics Dayananda Sagar College of Dental Sciences, Bengaluru Karnataka, India

Corresponding Author: NT Prashanth, Associate Professor Department of Oral and Maxillofacial Surgery, Dayananda Sagar College of Dental Sciences, Shavige Malleshwara Hills Kumaraswamy Layout, Bengaluru-560078, Karnataka, India e-mail: dr.n.t.prashanth@gmail.com
}

had significantly higher PTSD levels compared to male patients (at all the study intervals) and patients younger than 50 years of age had significantly higher PTSD levels compared to older patients.

Keywords: Facial injuries, Post-traumatic stress disorder, Trauma.

How to cite this article: Prashanth NT, Raghuveer HP, Kumar RD, Shobha ES, Rangan V, Hullale B. Post-traumatic Stress Disorder in Facial Injuries: A Comparative Study. J Contemp Dent Pract 2015;16(2):118-125.

\section{Source of support: Nil}

Conflict of interest: None

\section{INTRODUCTION}

The face or countenance plays an important part in the formation of initial social relationships and the appearance or 'attractiveness' of a person is greatly contributed by the face. ${ }^{1}$

The face can be disfigured secondarily by various causes; facial trauma is, however, the major cause for disfigurement.

Extensive bony and soft-tissue injuries of the face can lead to scarring or disfigurement of the facial region. Common sequelae of facial trauma patients are psychologic distress. Surgical and medical care of the trauma patients has progressed significantly over the last century and recent research has elaborated on the psychological aspects of traumatic events.

Our knowledge of the psychological impact of the traumatic events has historically been derived from the observation of military veterans or survivors of disasters. ${ }^{2}$ Maxillofacial trauma was recognized by Shepherd et $\mathrm{al}^{3}$ as an important target for research because of its potential for both physical and psychological disability.

The psychological aspects of maxillofacial trauma are poorly documented in routine clinical practice and unless it is recognized and treated; the psychological problems that arise post-trauma can become chronic.

There has been published data suggesting that posttraumatic stress disorder (PTSD) may develop and range between 26 and $41 \%$ of facial trauma victims. ${ }^{4-6}$

Post-traumatic stress disorder is defined in the fourth edition of the diagnostic and statistical manual of mental disorders (DSM-IV) ${ }^{7}$ as ${ }^{\prime}$ the development of characteristic 
symptoms following exposure to an extreme traumatic stress or involving direct personal experience of an event that involves actual or threatened death or serious injury or other threat to one's or another person's physical integrity'.

The symptoms include persistent re-experiencing of the traumatic event, nightmares, recurrent and intrusive recollections, avoidance of the situations with the traumatic event, sleeplessness and hyper vigilance. ${ }^{7}$ The symptoms must also be present for more than 1 month when symptoms are present between 1 and 3 months, PTSD is classified as acute and when symptoms last at least 3 months a chronic classification is given.

The aim of the study was to identify the presence of PTSD in patients who had sustained facial injuries; additionally, we aimed to identify other variables that may modify the psychological response to trauma that include gender, age and presence of disfigurement post- treatment and visible scars/orthopedic injuries.

\section{MATERIALS AND METHODS}

The subjects were recruited over a period of 30 months between December 2011 and July 2014, after approval had been obtained from the Institutional Ethical Committee.

Participants comprised of 460 patients from several multinodal trauma centers in Bengaluru city, Karnataka, who had sustained facial injuries that had healed either with or without significant disfigurement or scarring and with visible/orthopedic injuries. One hundred and eleven patients of the chosen 460 had sustained nondisfiguring facial injuries while 153 patients sustained disfiguring facial injuries; 64 patients who sustained facial injury (i.e. 19.5\%) were lost to follow-up and were not included in the study. One hundred and thirty-two had sustained orthopedic/visible injuries; however, in this group, 18 (i.e. $13.6 \%$ ) patients were lost to follow-up and were excluded from the study.

Grouping of the patients was done as follows:

- Patients with nondisfiguring facial injuries:

- Male: 54

- Female: 57

- Total: 111

- Patients with disfiguring facial injuries: Based on gender-

- Male: 81

- Female: 72

- Total: 153

- Patients with orthopedic/visible injuries:

- Male: 51

- Female: 63

- Total: 114
- Patients with disfiguring facial injuries: Based on age (total number of patients: 153)_

Male

- Less than 50 years: 51

- More than 50 years: 30

- Total: 81

Female

- Less than 50 years: 40

- More than 50 years: 32

- Total: 72

The inclusion criteria for those selected in the study were as follows:

- Age-18 years or older

- Glasgow coma scale on Admission-of 12 and above.

- Patients with facial injuries leading to scarring of $3 \mathrm{~cm}$ or more.

- Patients with facial injuries leading to facial disfigurement/asymmetry post-treatment.

- Patients with facial injuries with no disfigurement/ asymmetry or loss of form and function for a comparative study.

- Patients with a visible scar not covered by clothing on other parts of the body for a comparative study.

The exclusion criteria for those selected in the study were as follows:

- Patients with a history of alcohol dependence.

- Patients with a history of psychological dependence. The assessments were carried out at three time intervals [date of discharge (DOD), 1 month and 6 months postoperatively)] of the follow-up.

The impact of event scale (IES) ${ }^{8}$ was used to assess the PTSD; this is a widely used valid and reliable scale and provides for a low-cost short self-report measurement to detect PTSD. ${ }^{9}$

The IES scale consists of 15 items as 7 of which measure intrusive symptoms (intrusive thoughts, nightmares, intrusive feelings and imagery) and 8 of which measure avoidance symptoms (numbing of responsiveness, avoidance of feelings, situations and ideas) and both combined provide a subjective stress score. The responders to the scale rate the items on a four-point scale according to how often each of them occurred in the past 7 days. ${ }^{10}$

\section{IES Interpretation}

- 0-8: No meaningful impact.

- 9-25: Impact event - you may be affected.

- 26-43: Powerful impact event - you are certainly affected.

- 44-75: Severe impact event - this is capable of altering your ability of function. 


\section{Score Consequence}

- Twenty-seven or more: There is a $75 \%$ chance that the patient has PTSD. ${ }^{11}$ Those who do not have full PTSD may have partial PTSD or at least some of the symptoms.

- Thirty-five and above: This represents the best cut-off for a probable diagnosis of PTSD $^{12}$ — a mental health professional skilled to treat such issues should be consulted.

\section{RESULTS}

\section{Statistical Analysis}

The statistical analysis was done by Mann-Whitney Utest for determining and comparing the impact of event scores among the patients and the following results were obtained.

Comparison of IES scores between nondisfiguring facial and facial disfiguring injuries (Table 1):

- In patients with nondisfiguring facial injuries the mean score of IES at the DOD was $32.05( \pm 7.87)$ compared to patients with disfiguring facial injuries mean score $48.39( \pm 10.29)$ and this was statistically significant.

- In patients with nondisfiguring facial injuries, the mean score of IES at discharge after 1 month was
$6.17( \pm 2.17)$ compared to patients with disfiguring facial injuries mean score $35.16( \pm 12.08)$ and this was statistically significant.

- In patients with nondisfiguring facial injuries, the mean score of IES at discharge after 6 months was $2.07( \pm 0.37)$ compared to patients with disfiguring facial injuries mean score $29.02( \pm 13.49)$ and this was statistically significant.

Comparison of IES scores between nondisfiguring facial and facial disfiguring injuries (males) (Table 2):

- In comparative study between facial nondisfiguring injuries of male patients the mean score of IES of the patients at the DOD was $24.93( \pm 5.02)$ and when compared to patients with disfiguring facial injuries the mean score $42.26( \pm 5.16)$ and this was statistically significant.

- In comparative study between facial nondisfiguring injuries of male patients the mean score of IES after 1 month was $4.56( \pm 1.08)$ and when compared to patients with disfiguring facial injuries the mean score 28.15 ( \pm 8.12$)$ and this was statistically significant.

- In comparative study between facial nondisfiguring injuries of male patients, the mean score of IES after 6 months was $2.00( \pm 0.00)$ and when compared to patients with disfiguring facial injuries the mean score $19.85( \pm 4.46)$ and this was statistically significant.

Table 1: Comparison between nondisfiguring and facial disfiguring injuries

\begin{tabular}{|c|c|c|c|c|c|c|c|c|c|}
\hline Scale & Time interval & Injury & $n$ & Mean & $S D$ & $S D$ & Mean difference & z-value & $p$-value \\
\hline \multirow[t]{6}{*}{ Impact of events } & DOD & $\begin{array}{l}\text { Facial non- } \\
\text { disfiguring }\end{array}$ & 111 & 32.05 & 7.87 & 0.75 & -16.341 & -11.404 & $<0.001^{*}$ \\
\hline & & $\begin{array}{l}\text { Facial } \\
\text { disfiguring }\end{array}$ & 153 & 48.39 & 10.29 & 0.83 & & & \\
\hline & 1 month & $\begin{array}{l}\text { Facial non- } \\
\text { disfiguring }\end{array}$ & 111 & 6.17 & 2.17 & 0.21 & -28.992 & -14.034 & $<0.001^{*}$ \\
\hline & & $\begin{array}{l}\text { Facial } \\
\text { disfiguring }\end{array}$ & 153 & 35.16 & 12.08 & 0.98 & & & \\
\hline & 6 months & $\begin{array}{l}\text { Facial non- } \\
\text { disfiguring }\end{array}$ & 111 & 2.07 & 0.37 & 0.04 & -26.948 & -14.300 & $<0.001^{*}$ \\
\hline & & $\begin{array}{l}\text { Facial } \\
\text { disfiguring }\end{array}$ & 153 & 29.02 & 13.49 & 1.09 & & & \\
\hline
\end{tabular}

Table 2: Comparison between nondisfiguring injuries and facial disfiguring injuries (males)

\begin{tabular}{|c|c|c|c|c|c|c|c|c|c|}
\hline Scale & Time interval & Injury & $n$ & Mean & STD Dev & SE of mean & Mean difference & z-value & $p$-value \\
\hline \multirow[t]{6}{*}{ Impact of events } & DOD & $\begin{array}{l}\text { Facial non- } \\
\text { disfiguring }\end{array}$ & 54 & 24.93 & 5.02 & 0.68 & -17.333 & -10.247 & $<0.001^{*}$ \\
\hline & & $\begin{array}{l}\text { Facial } \\
\text { disfiguring }\end{array}$ & 81 & 42.26 & 5.16 & 0.57 & & & \\
\hline & 1 month & $\begin{array}{l}\text { Facial non- } \\
\text { disfiguring }\end{array}$ & 54 & 4.56 & 1.08 & 0.15 & -23.593 & -10.203 & $<0.001^{*}$ \\
\hline & & $\begin{array}{l}\text { Facial } \\
\text { disfiguring }\end{array}$ & 81 & 28.15 & 8.12 & 0.90 & & & \\
\hline & 6 months & $\begin{array}{l}\text { Facial non- } \\
\text { disfiguring }\end{array}$ & 54 & 2.00 & 0.00 & 0.00 & -17.852 & -10.339 & $<0.001^{*}$ \\
\hline & & $\begin{array}{l}\text { Facial } \\
\text { disfiguring }\end{array}$ & 81 & 19.85 & 4.46 & 0.50 & & & \\
\hline
\end{tabular}


Comparison of IES scores between nondisfiguring facial and facial disfiguring injuries (females) (Table 3):

- In comparative study between facial nondisfiguring injuries of female patients, the mean score of IES at the DOD was $38.79( \pm 1.66)$ and female patients with disfiguring facial injuries the mean score was 55.28 $( \pm 10.29)$ and this was statistically significant.

- In comparative study between facial nondisfiguring injuries of female patients, the mean score of IES after 1 month was $7.70( \pm 1.79)$ and female patients with disfiguring facial injuries the mean score was 43.06 $( \pm 10.29)$ and this was statistically significant.

- In comparative study between facial non-disfiguring injuries of female patients the mean score of IES after 6 months was $2.14( \pm 0.52)$ and female patients with disfiguring facial injuries the mean score was 39.33 $( \pm 12.79)$ and this was statistically significant.

Comparison of IES scores among patients with disfiguring facial injuries, nondisfiguring facial injuries and orthopedic/visible scarring (Table 4):

- In patients with orthopedic/visible injuries at the DOD, the mean IES score was $9.54 \pm 4.32$ compared to patients who had facial nondisfiguring injuries, where the mean IES was $32.05( \pm 7.87)$ and, in patients with disfiguring facial injuries, the mean IES score was $48.39( \pm 10.29)$, and this was statistically significant $(\mathrm{p}<0.001)$.
- In patients with orthopedic/visible injuries after 1 month the mean IES score was $2.84( \pm 5.76)$ compared to patients who had facial nondisfiguring injuries where the mean IES was $6.17( \pm 2.17)$ and, in patients with disfiguring facial injuries, the mean IES score was $35.16( \pm 12.08)$ and this was statistically significant $(\mathrm{p}<0.001)$.

- In patients with orthopedic/visible injuries after 6 months, the mean IES score was $1.68( \pm 3.31)$ compared to patients who had facial nondisfiguring injuries where the mean IES was $2.07( \pm 0.37)$ and in patients with disfiguring facial injuries the mean IES score was $29.02( \pm 13.49)$ and this was statistically significant $(\mathrm{p}<0.001)$.

Comparison of IES scores among male and female patients with orthopedic/visible scarring (Table 5):

- In patients with orthopedic/visible injuries at the DOD, the mean IES score of male patients was 9.53 $( \pm 6.43)$ compared to female patients who had a mean score of $9.38( \pm 0.79)$ at the DOD and this was statistically significant while there was no significant difference observed at 1 month and 6 months postoperatively.

Comparison of IES scores among female and male patients with facial injuries disfiguring (Table 6):

- In patients with facial disfiguring injuries the mean score of IES of female patients on DOD was higher

Table 3: Comparison between nondisfiguring and facial disfiguring injuries (females)

\begin{tabular}{|c|c|c|c|c|c|c|c|c|c|}
\hline Scale & Time interval & Injury & $n$ & Mean & $S D$ & SE of mean & Mean difference & z-value & $p$-value \\
\hline \multirow[t]{6}{*}{ Impact of events } & DOD & $\begin{array}{l}\text { Facial non- } \\
\text { disfiguring }\end{array}$ & 57 & 38.79 & 1.66 & 0.22 & -16.488 & -10.000 & $<0.001^{*}$ \\
\hline & & $\begin{array}{l}\text { Facial } \\
\text { disfiguring }\end{array}$ & 72 & 55.28 & 10.28 & 1.21 & & & \\
\hline & 1 month & $\begin{array}{l}\text { Facial non- } \\
\text { disfiguring }\end{array}$ & 57 & 7.70 & 1.79 & 0.24 & -35.354 & -10.000 & $<0.001^{*}$ \\
\hline & & $\begin{array}{l}\text { Facial } \\
\text { disfiguring }\end{array}$ & 72 & 43.06 & 10.90 & 1.28 & & & \\
\hline & 6 months & $\begin{array}{l}\text { Facial non- } \\
\text { disfiguring }\end{array}$ & 57 & 2.14 & 0.52 & 0.07 & -37.193 & -10.000 & $<0.001^{*}$ \\
\hline & & $\begin{array}{l}\text { Facial } \\
\text { disfiguring }\end{array}$ & 72 & 39.33 & 12.79 & 1.51 & & & \\
\hline
\end{tabular}

Table 4: Comparison between nondisfiguring, facial disfiguring and orthopedic/visible injuries

\begin{tabular}{|c|c|c|c|c|c|c|c|c|}
\hline Scale & Time interval & Injury & $N$ & Mean & $S D$ & Min & Max & $p$-value \\
\hline \multirow[t]{9}{*}{ Impact of events } & \multirow[t]{3}{*}{ DOD } & Nondisfiguring & 111 & 32.05 & 7.87 & 13.00 & 40 & \multirow[t]{3}{*}{$<0.001^{*}$} \\
\hline & & Disfiguring & 153 & 48.39 & 10.29 & 36.00 & 66 & \\
\hline & & Orthopedic & 114 & 9.45 & 4.32 & 6.00 & 21 & \\
\hline & \multirow[t]{3}{*}{1 month } & Nondisfiguring & 111 & 6.17 & 2.17 & 2.00 & 11 & \multirow[t]{3}{*}{$<0.001^{*}$} \\
\hline & & Disfiguring & 153 & 35.16 & 12.08 & 15.00 & 55 & \\
\hline & & Orthopedic & 114 & 2.84 & 5.76 & 0.00 & 17 & \\
\hline & \multirow[t]{3}{*}{6 months } & Nondisfiguring & 111 & 2.07 & 0.37 & 1.00 & 3 & \multirow[t]{3}{*}{$<0.001^{*}$} \\
\hline & & Disfiguring & 153 & 29.02 & 13.49 & 10.00 & 53 & \\
\hline & & Orthopedic & 114 & 1.68 & 3.31 & 0.00 & 9 & \\
\hline
\end{tabular}


(55.28 \pm 10.28$)$ compared to male patients $(42.26 \pm 5.16)$ and this difference was statistically significant $(\mathrm{p}<$ 0.001 ).

- In patients with facial disfiguring injuries, the mean score of IES of female patients after 1 month of discharge was higher $(43.06 \pm 10.90)$ compared to male patients $(28.15 \pm 8.12)$ and this difference was statistically significant $(\mathrm{p}<0.001)$.

- In patients with facial disfiguring injuries, the mean score of IES of female patients after 6 months of discharge was higher $(39.33 \pm 12.79)$ compared to male patients $(19.85 \pm 4.46)$ and this difference was statistically significant $(\mathrm{p}<0.001)$.

Comparison of IES scores in male patients with facial injuries disfiguring (male patients) (Table 7):

- The mean score of IES of male patients with facial injuries at the DOD was higher in less than 50 years $(45.94 \pm 2.31)$ age group compared to patients aged more than 50 years $(36.00 \pm 0.00)$ and this difference was statistically significant $(\mathrm{p}<0.001)$.

- The mean score of IES of male with facial injuries after 1 month of discharge was higher in less than 50 years $(34.12 \pm 1.93)$ age group compared to more than 50 years $(18.00 \pm 2.49)$ and this difference was statistically significant $(\mathrm{p}<0.001)$.

- The mean score of IES of male patients after 6 months of discharge was higher in less than 50 years (22.47 \pm
1.16) age group compared to more than 50 years $(15.40 \pm$ $4.48)$ and this difference was statistically significant $(\mathrm{p}<0.001)$.

Comparison of IES scores in female patient with facial injuries disfiguring (female patients) (Table 8):

- The mean score of IES of female patients with facial injuries on DOD was higher in less than 50 years $(63.50 \pm 5.76)$ age group compared to more than 50 years age group $(45.00 \pm 2.03)$ and this difference was statistically significant $(\mathrm{p}<0.001)$.

- The mean score of IES of female patients with facial injuries on discharge after 1 month was higher in less than 50 years $(51.10 \pm 7.99)$ age group compared to more than 50 years age group $(33.00 \pm 2.03)$ and this difference was statistically significant $(\mathrm{p}<0.001)$.

- The mean score of IES of female patients with facial injuries discharge after 6 months was higher in less than 50 years age group $(49.20 \pm 7.74)$ compared to more than 50 years age group $(27.00 \pm 4.06)$ and this difference was statistically significant $(p<0.001)$.

Comparison of IES scores in female and male patients with facial injuries disfiguring (age group less than 50 years of age) (Table 9):

- The mean score of IES on DOD was higher in less than 50 years $(63.50 \pm 5.76)$ age group female patients compared to male patients $(45.94 \pm 2.31)$ and this difference was statistically significant $(\mathrm{p}<0.001)$.

Table 5: Comparison between male and female orthopedic/visible patients

\begin{tabular}{|c|c|c|c|c|c|c|c|c|c|}
\hline Scale & Time interval & Gender & $N$ & Mean & $S D$ & SE of mean & Mean difference & z-value & $p$-value \\
\hline \multirow[t]{6}{*}{ Impact of events } & DOD & Male & 51 & 9.53 & 6.43 & 0.90 & 0.148 & -5.202 & $<0.001^{*}$ \\
\hline & & Female & 63 & 9.38 & 0.79 & 0.10 & & & \\
\hline & 1 month & Male & 51 & 4.00 & 7.28 & 1.02 & 2.095 & -1.154 & 0.248 \\
\hline & & Female & 63 & 1.90 & 3.96 & 0.50 & & & \\
\hline & 6 months & Male & 51 & 1.65 & 3.00 & 0.42 & -0.067 & 0.000 & 1.000 \\
\hline & & Female & 63 & 1.71 & 3.56 & 0.45 & & & \\
\hline
\end{tabular}

Table 6: Comparison between male and female facial injuries disfiguring

\begin{tabular}{llllllllll}
\hline Scale & Time interval & Gender & $N$ & Mean & SD & SE of mean & Mean difference & z-value & $p$-value \\
\hline Impact of events & DOD & Male & 81 & 42.26 & 5.16 & 0.57 & -13.019 & -6.925 & $<0.001^{*}$ \\
& & Female & 72 & 55.28 & 10.28 & 1.21 & & -6.905 & $<0.001^{*}$ \\
& \multirow{2}{*}{1 month } & Male & 81 & 28.15 & 8.12 & 0.90 & -14.907 & & \\
& \multirow{4}{*}{6 months } & Female & 72 & 43.06 & 10.90 & 1.28 & & -9.766 & $<0.001^{*}$ \\
& & Male & 81 & 19.85 & 4.46 & 0.50 & -19.481 & & \\
& & Female & 72 & 39.33 & 12.79 & 1.51 & & & \\
\hline
\end{tabular}

Table 7: Comparison between less than 50 and more than 50 years age group disfiguring facial injuries (male patients)

\begin{tabular}{|c|c|c|c|c|c|c|c|c|c|}
\hline Scale & Time interval & Age group & $N$ & Mean & $S D$ & SE of mean & Mean difference & z-value & $p$-value \\
\hline \multirow[t]{6}{*}{ Impact of events } & DOD & $<50$ years & 51 & 45.94 & 2.31 & 0.32 & 9.941 & -8.321 & $<0.001^{*}$ \\
\hline & & $>50$ years & 30 & 36.00 & 0.00 & 0.00 & & & \\
\hline & 1 month & $<50$ years & 51 & 34.12 & 1.93 & 0.27 & 16.118 & -8.138 & $<0.001^{*}$ \\
\hline & & $>50$ years & 30 & 18.00 & 2.49 & 0.45 & & & \\
\hline & 6 months & $<50$ years & 51 & 22.47 & 1.16 & 0.16 & 7.071 & -8.138 & $<0.001^{*}$ \\
\hline & & $>50$ years & 30 & 15.40 & 4.48 & 0.82 & & & \\
\hline
\end{tabular}


- The mean score of IES on discharge after 1 month was higher in less than 50 years $(51.10 \pm 7.99)$ age group female patients compared to male patients (34.12 \pm 1.93) and this difference was statistically significant $(\mathrm{p}<0.001)$.

- The mean score of IES on DOD after 6 months was higher in less than 50 years $(49.20 \pm 7.74)$ age group compared to male patients $(22.47 \pm 1.16)$ and this difference was statistically significant $(\mathrm{p}<0.001)$.

Comparison of IES scores in female and male patient with facial injuries disfiguring (age group more than 50 years of age) (Table 10):

- The mean score of IES on DOD was higher in female patients more than 50 years $(45.00 \pm 2.03)$ age group compared to male patients aged more than 50 years $(36.00 \pm 0.00)$ and this difference was statistically significant $(\mathrm{p}<0.001)$.

- The mean score of IES on DOD after 1 month was higher in female patients more than 50 years $(33.00 \pm$ 2.03) age group compared to male patients aged more than 50 years $(36.00 \pm 0.00)$ and this difference was statistically significant $(\mathrm{p}<0.001)$.

- The mean score of IES on DOD after 6 months was higher in female patients more than 50 years $(27.00 \pm$ 4.06) age group compared to male patients aged more than 50 years $(15.40 \pm 4.48)$ and this difference was statistically significant $(\mathrm{p}<0.001)$.

\section{DISCUSSION}

Research in trauma is increasingly being done to identify predictors of the psychological morbidity that follows. Earlier studies have established the presence of PTSD in patients with facial injuries. ${ }^{4,13,14}$ There is not much information available on PTSD levels in patients who had sustained facial trauma with disfigurement/scarring post operatively and patients who had facial injuries which had healed without disfigurement/scarring.

The present study has shown statistically significant increased IES scores in patients who had suffered disfiguring facial injuries compared to patients whose facial injuries had healed without disfigurement. This increased scores were seen at all three time intervals (DOD, 1 month and 6 months postoperatively). Significant higher scores of IES was also seen when the data was broken up and compared between the male patients who had sustained disfiguring facial injuries and those who had nondisfiguring facial injuries and also between female patients with facial disfiguring injuries and nondisfiguring facial injuries.

These results indicate the presence of PTSD in patients who had sustained facial disfiguring injuries while those who had nondisfiguring facial injuries had some psychological morbidity but could not be considered to be PTSD and also there were significantly higher PTSD levels in females compared to the male patients.

Table 8: Comparison between less than 50 and more than 50 years age group disfiguring facial injuries (female patients)

\begin{tabular}{|c|c|c|c|c|c|c|c|c|c|}
\hline Scale & Time interval & Age group & $N$ & Mean & $S D$ & SE of mean & Mean difference & z-value & $p$-value \\
\hline \multirow[t]{6}{*}{ Impact of events } & DOD & $<50$ yrs & 40 & 63.50 & 5.76 & 0.91 & 18.500 & -7.131 & $<0.001^{*}$ \\
\hline & & $>50 \mathrm{yrs}$ & 32 & 45.00 & 2.03 & 0.36 & & & \\
\hline & 1 month & $<50$ yrs & 40 & 51.10 & 7.99 & 1.26 & 18.100 & -7.131 & $<0.001^{*}$ \\
\hline & & $>50$ yrs & 32 & 33.00 & 2.03 & 0.36 & & & \\
\hline & 6 months & $<50$ yrs & 40 & 49.20 & 7.74 & 1.22 & 22.200 & -7.516 & $<0.001^{*}$ \\
\hline & & $>50 \mathrm{yrs}$ & 32 & 27.00 & 4.06 & 0.72 & & & \\
\hline
\end{tabular}

Table 9: Comparison between male and female disfiguring facial patients (age group less than 50 years of age)

\begin{tabular}{llllllllll}
\hline Scale & Time interval & Gender & $N$ & Mean & SD & SE of mean & Mean difference & z-value & $p$-value \\
\hline Impact of events & DOD & Male & 51 & 45.94 & 2.31 & 0.32 & -17.559 & -8.085 & $<0.001^{*}$ \\
& & Female & 40 & 63.50 & 5.76 & 0.91 & & -8.085 & $<0.001^{*}$ \\
& \multirow{4}{*}{ 1 month } & Male & 51 & 34.12 & 1.93 & 0.27 & -16.982 & & \\
& \multirow{4}{*}{6 months } & Female & 40 & 51.10 & 7.99 & 1.26 & & -8.810 & $<0.001^{*}$ \\
& & Male & 51 & 22.47 & 1.16 & 0.16 & -26.729 & & \\
& & Female & 40 & 49.20 & 7.74 & 1.22 & & & \\
\hline
\end{tabular}

Table 10: Comparison between male and female disfiguring facial patients (age group more than 50 years of age)

\begin{tabular}{|c|c|c|c|c|c|c|c|c|c|}
\hline $\begin{array}{l}\text { Scale } \\
\text { Impact of events }\end{array}$ & Time interval & Gender & $N$ & Mean & $S D$ & SE of mean & Mean difference & z-value & $p$-value \\
\hline \multirow[t]{6}{*}{ Impact of events } & \multirow[t]{2}{*}{ DOD } & Male & 30 & 36.00 & 0.00 & 0.00 & \multirow[t]{2}{*}{-9.000} & \multirow[t]{2}{*}{-7.323} & \multirow[t]{2}{*}{$<0.001^{*}$} \\
\hline & & Female & 32 & 45.00 & 2.03 & 0.36 & & & \\
\hline & \multirow[t]{2}{*}{1 month } & Male & 30 & 18.00 & 2.49 & 0.45 & \multirow[t]{2}{*}{-15.000} & \multirow[t]{2}{*}{-6.995} & \multirow[t]{2}{*}{$<0.001^{*}$} \\
\hline & & Female & 32 & 33.00 & 2.03 & 0.36 & & & \\
\hline & \multirow[t]{2}{*}{6 months } & Male & 30 & 15.40 & 4.48 & 0.82 & \multirow[t]{2}{*}{-11.600} & \multirow[t]{2}{*}{-6.995} & \multirow[t]{2}{*}{$<0.001^{*}$} \\
\hline & & Female & 32 & 27.00 & 4.06 & 0.72 & & & \\
\hline
\end{tabular}


Though PTSD cannot be diagnosed within 1 month of the incident, we strived to try and identify patients with psychological problems within the DOD (usually within a week) and study them prospectively over 1 and 6 months periods.

Earlier studies have mentioned that the female patients were associated with significantly higher anxiety and depression scores following facial injury when compared to men ${ }^{15}$ our study tried to compare the PTSD levels among male and female patients who had facial injuries that led to disfigurement.

The mean scores of IES of female patients with facial disfiguring injuries were significantly higher than male patients at all three study intervals (DOD, 1 month and 6 months) these results implied both the groups had PTSD and that female patients had significantly higher PTSD levels compared to male patients; this concurs with other studies where the female patients were associated with higher psychological morbidity after a traumatic incident.

As appearance or 'attractiveness' is associated with the facial region, an important aspect we wanted to study was presence of PTSD in patients with a visible injury not covered by clothing like an injury on the hand/ orthopedic injury and facial injuries.

Our study found that there was a highly significant increase in the IES scores of patients who had sustained disfiguring facial injuries compared to visible scarring/ orthopedic injuries and this was seen in all the study intervals (DOD, 1 and 6 months). This established that higher PTSD levels were seen in patients with disfiguring facial injuries compared to other body scars/disfigurement.

Another important variable we set to find out was if age of the patients sustaining facial injuries was a factor in increased PTSD levels.

The present study found that both the male and female patients sustaining disfiguring facial injuries and who were less than 50 years of age had higher IES scores compared to patients who were more than 50 years of age. This shows that age is a significant factor in the development of PTSD in patients who had sustained disfiguring facial injuries and also on comparison the female patients who were less than 50 years of age had higher PTSD levels compared to male patients who were less than 50 years of age.

Our study concurs that psychological disturbances present at the time of discharge and which were at a high level persisted at a high level throughout the follow-up period. It also confirms that serious attempts have to be made to try and identify these at-risk patients at an earlier stage to provide psychiatric counseling and treatment so that comprehensive care of the facial trauma patient can be carried out.

Further, PTSD has been associated with a chronic disturbance in homeostasis ${ }^{16}$ which might lead to a prolonged inflammatory period and so healing time of facial injury patients might be protracted.

Taking all this into consideration, it is important that the nursing, para-medical and the surgical staff have an awareness of the psychological morbidity that a facial injury patient might develop, one study has discussed that this knowledge is limited only to the staff of trauma units. ${ }^{17}$

Our study has provided further information on PTSD in facial injuries and provides yet another reason to the clinician that early evaluation and treatment may prevent or lessen the PTSD levels. Further investigations on the psychological morbidity accompanying facial trauma is clearly needed.

\section{CONCLUSION}

The results of our study led to the conclusion that in comparison with patients who had nondisfiguring facial injuries and disfiguring facial injuries, the mean IES scores were significantly higher in the disfiguring facial injury patients, this implied increased PTSD levels and this was observed both in the male and female patients and at all the three study intervals (DOD, 1 and 6 months). The mean IES scores of patients with orthopedic/visible injuries was significantly less and could not be termed strictly as PTSD and when compared to facial disfiguring injury patients the mean IES was significantly higher corresponding to higher levels of PTSD and this may be because the face is perceived for its role in 'attractiveness'/ appearance of the individual. We also observed the mean scores of IES was higher in females compared to males in all the study groups, implying higher PTSD levels in females when injuries affect the face and also age contributed to higher means of IES and corresponding higher levels of PTSD with both the male and female facial injury patients who aged less than 50 years having significant higher means of IES than patients above 50 years of age and this was statistically significant in all the three intervals of study (DOD, 1 and 6 months postoperatively).

\section{CLINICAL SIGNIFICANCE}

Early recognition of at-risk individuals who might develop PTSD symptoms that go on to become chronic is essential for the comprehensive management of the facial trauma patient. In our study, we found that injuries to the face leading to disfigurement, gender (female) and age (less than 50 years) had increased PTSD levels. 
Clinically, facial injury patients can be given structured self-report questionnaires to help us to identify and predict the presence of psychological morbidity that develops in some individuals and lead to PTSD later on, after an early assessment, the treatment of such morbidities can be undertaken by staff skilled to manage such issues, this leads to faster recovery and improvement in the quality of life of the facial injury patients.

\section{REFERENCES}

1. Tavane PN, et al. Validation of a Kannada version of the Impact of Events Scale. J Int Oral Health 2013 Sept-Oct; 5(5):38-47.

2. Alexander DA. Human reactions to trauma: their features and management. In: Greaves I, Porter K. editors. Pre-hospital medicine: the principles and practice of immediate care. Arnold, London; 1999.

3. Shepherd JP. Strategies for the study of long-term sequelae of oral and facial injuries. Journal of Oral and Maxillofacial Surgery 1992;50:390-399.

4. Bisson JI, Shepherd JP, Dhutia M. Psychological sequelae of facial trauma. Journal of Trauma 1997;43:496-500.

5. Hull AM, Lowe T, Devlin M, Finlay P, Koppel D, Stewart AM. Psychological Sequelae of maxillofacial trauma: a preliminary study. Br J Oral Maxillofac Surg 2003;41:317-322.

6. Roccia F, Dell'Acqua A, Angelini G, Berrone S. Maxillofacial trauma and psychiatric sequelae: post-traumatic stress disorder. The Journal of craniofacial surgery 2005;16(3):355-360.

7. Diagnostic and Statistical Model of Mental Disorders. 4th ed. Washington (DC). American Psychiatric Association; 1994.
8. Horowitz M, Wilner N, Alvarez W. Impact of Events Scale: A measure of subjective stress. Psychosom Med 1979;41(3): 209-218.

9. Rothbaum BO, Foa EB, Riggs DS, Murdock TB, Walsh W. A prospective examination of posttraumatic stress disorder in rape victims. Journal of Traumatic Stress 1992;5:455-475.

10. Devilly GJ, Spence SH. The relative efficacy and treatment distress of EMDR and a cognitive-behavior trauma treatment protocol in the amelioration of posttraumatic stress disorder. Journal of Anxiety Disorders 1999;13:131-157.

11. Coffey SF, Berglind G, Screening for PTSD in motor vehicle accident survivors using PSS-SR and IES. Journal of Traumatic Stress 2006;19(1):119-128.

12. Neal LA, Walter B, Rollins J, et al. Convergent Validity of Measures of Post-Traumatic Stress Disorder in a Mixed Military and Civilian Population. Journal of Traumatic Stress 1994;7(3):447-455.

13. Sen P, Ross N, Rogers S. Recovering maxillofacial trauma patients: the hidden problems. J Wound Care 2001;10:53e57.

14. Hull AM, Lowe T, Finlay PM. The psychological impact of maxillofacial trauma: an overview of reactions to trauma. Oral Surgery, Oral Medicine, Oral Pathology, Oral Radiology and Endodontics 2003;95(5):515-520.

15. Islam S, Ahmed M, Walton GM. The association between depression and anxiety disorders following facial trauma-a comparative study. Injury 2010;41(1):92-96.

16. Miller RJ, Sutherland AG, Hutchison JD, Alexander DA. C-reactive protein and interleukin 6 receptor in posttraumatic stress disorder: a pilot study. Cytokine 2001;13(4): 253-255.

17. Alexander DA, Klein S. The epidemiology of PTSD and patient vulnerability factors. Psychiatry 2003;2:22-25. 